\title{
Grain Boundary Structure Reconstruction due toVacancies and Dopants in Oxides
}

\author{
Y. Ikuhara ${ }^{1,2,3}$ \\ ${ }^{1}$ Institute of Engineering Innovation, The University of Tokyo, Tokyo, 113-8656, Japan \\ ${ }^{2}$ Nanostructures Research Laboratory, Japan Fine Ceramics Center, Nagoya, 456-8587, Japan \\ ${ }^{3}$ WPI-AIMR Research Center, Tohoku University, Sendai, 980-8577, Japan
}

Mechanical and functional properties of materials are strongly dependent on the grain boundaries (GBs) which has different atomic structures due to the disorder in periodicity. The grain boundary structures are also influenced by the dopant and vacancies segregated at GBs, providing the functional properties, which cannot be observed in a perfect crystal. In order to control GB structures to improve the properties, we need to understand the relationships between GB characters, atomic structures and chemistry. It is considered that the formation of vacancies reconstructs the GB atomic structures, depending on the GB characters such as misorientation angle and GB plane. In addition, the segregated dopants also should play an important role to reconstruct the GB structures, which is related to the GB structural transition.

$\mathrm{CeO}_{2}$ has attracted much attention as electrolyte materials for solid oxide fuel cells. It has been reported that GBs play an important role in the oxygen transport properties in $\mathrm{CeO}_{2}$, which must be influenced by the vacancies introduced in GBs. It has also been known that a small amount of praseodymium (Pr) dopant is very effective to improve the varistic property of $\mathrm{ZnO}$, which is related to the Pr segregation at the GBs. In this study, well-defined GBs structures of $\mathrm{CeO}_{2}$ and $\mathrm{ZnO}$, which are fabricated by the bicrystal techniques [1-3], are used as the model samples, and the behavior of the GB structure reconstruction due to vacancies and dopants are systematically investigated by combining aberrationcorrected STEM, EELS and theoretical calculations. STEM observations were performed using JEM2100F and ARM-200F (JEOL) equipped with CEOS Cs-corrector. EELS spectra were acquired in STEM mode by an Enfina spectrometer (Gatan Inc). For theoretical approach, static lattice and density functional theory (DFT) calculations were used complementary.

Fig.1(a) shows HAADF STEM image of $\Sigma 5(210) /[001]$ GB [1], in which the periodic structural units are shown. In the figure, (c) the most stable stoichiometric GB structure and (b) simulated STEM images based on the model (c) are shown. As can be seen, the experimental image is not consistent with the theoretical image. But, both images are found to be consistent, comparing (e) the theoretically obtained most stable non-stoichiometric GB structure and (f) the corresponding simulated STEM images. In the case of $\Sigma 5 \mathrm{~GB}$, nonstoichiometric GB core structure with oxygen vacancies is considered to be the most suitable model for the experimentally observed $\Sigma 5 \mathrm{~GB}$. On the other hand, the $\Sigma 3$ GB has the stoichiometric GB core structure [2]. According to the calculations, the structural distortions at the $\Sigma 3 \mathrm{~GB}$ are not as clear as those at the $\Sigma 5$ stoichiometric GB. These results suggest that the oxygen stoichiometry at the GBs does not only depend on the atmosphere but also on the GB atomic structure, which is closely related to the GB energies, dangling bonds and strain.

Fig.2 (a) shows (a) HAADF STEM image of the Pr-doped ZnO $\Sigma 13\left(0=27.8^{\circ}\right) \mathrm{GB}$, indicating that GB plane is parallel to (2570), and the zig-zag alignment of structural units (SUs) are formed. This SU is named as $\gamma \mathrm{SU}$, comparing to the $\alpha$ and $\beta$ SUs appeared in another GBs with smaller $\theta$ [3]. As can be 
seen in the figure, the Pr segregation sites form trimmer-like configuration. The dotted lines show a structural period, and set of circles denote the atomic arrangement in a single period. Fig.2 (b) shows the most stable atomic configuration obtained by the first-principles calculation. The structure contains zigzag-aligned two SUs per a single period. These SUs are categorized into SU $\gamma$, where three columns are occupied with Pr. The trimmer-like structure directs inversely for the neighboring SUs, resulting in the zigzag feature. Location of $\mathrm{Pr}$ is consistent with the location observed in the STEM image. In the presentation, it will be discussed that the structure of Pr-doped GBs has different structural sequence with the increase of $\theta$. For smaller $\theta$, Pr simply substitutes particular columns in SUs $\alpha$ and/or $\beta$ without causing transition to SU $\gamma$. For higher $\theta\left(\theta=27.8^{\circ}\right)$, structure changes into SU $\gamma$. It is thus concluded that GB structural transition occurs at proper $\theta$ degree for the [0001] symmetrical tilt GBs.

\section{Acknowledgements}

This work was collaborated with B. Feng, H. Hojo, J-Y. Roh, Y. Sato, H.Ohta, T. Mizoguchi, N. Shibata, and supported in part by the Grant-in-Aid for Scientific Research on Innovative Areas "Nano Informatics" (Grant No. 25106003) from JSPS and "Nanotechnology Platform" (Project No. 12024046) of MEXT, Japan.

\section{References}

[1] H. Hojo, T. Mizoguchi, H. Ohta, S.D. Findlawy, N. Shibata, T. Yamamoto and Y. Ikuhara, Nano Lett, 10, 4668 (2010).

[2] B. Feng, H. Hojo, T. Mizoguchi, H. Ohta, S.D. Findlay, Y. Sato, N. Shibata, T. Yamamoto and Y. Ikuhara, Appl Phys Lett, 100, 073109 (2012).

[3] Y. Sato, J-Y. Roh, Y. Ikuhara, Phys. Rev. B, 87, 140101 (2013)
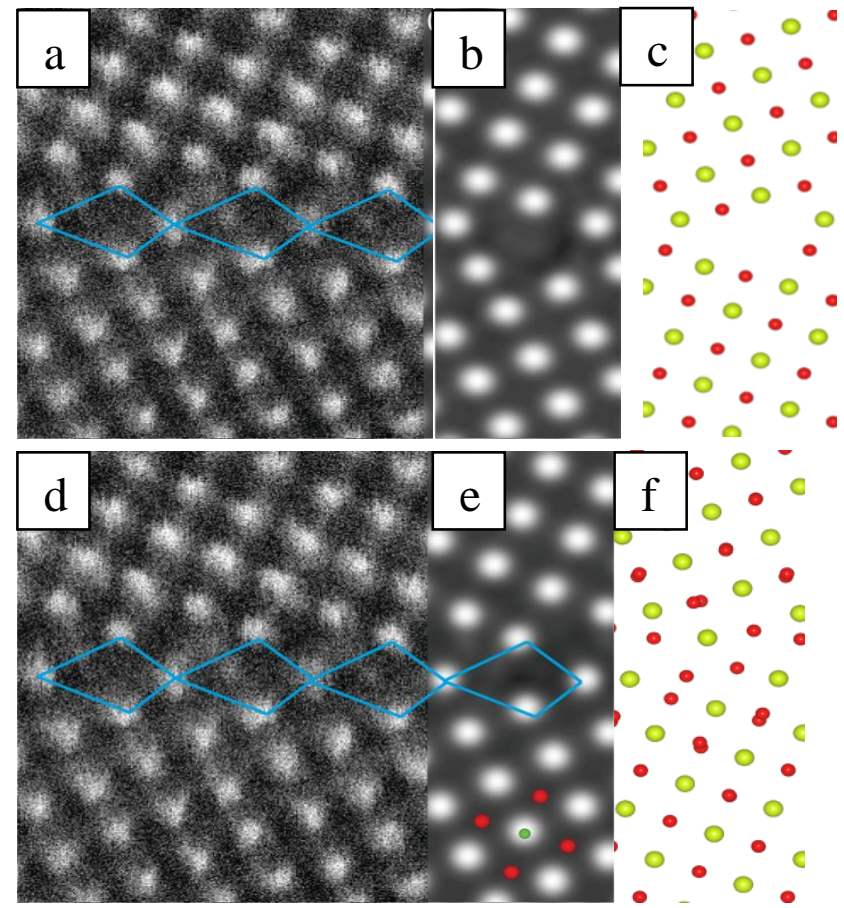

Fig.1: HAAD F STEM images, simulation images, and the calculated GB structures for $\Sigma 5(210) /[001] \mathrm{GB}$ with (a-c) stoichiometric and (d-f) non-stoichiometric models.

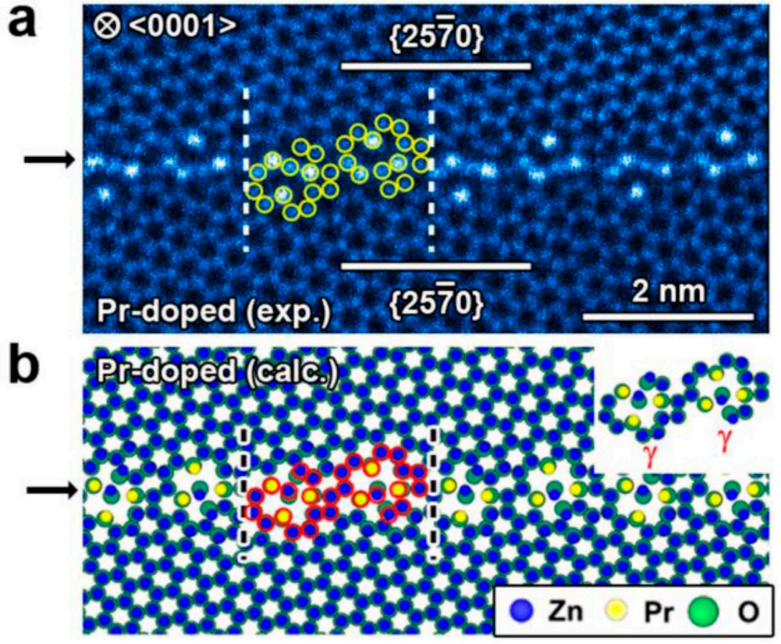

Fig.2: (a) HAADF STEM image of Pr-doped $\mathrm{ZnO} \Sigma 13\left(\theta=27.8^{\circ}\right)$ and (b) most stable atomic configuration obtained by the first-principles calculations. The dotted lines show a structural period, and set of circles denote the atomic arrangement in a single period. SUs $(\gamma \gamma)$ are shown in the inset. 